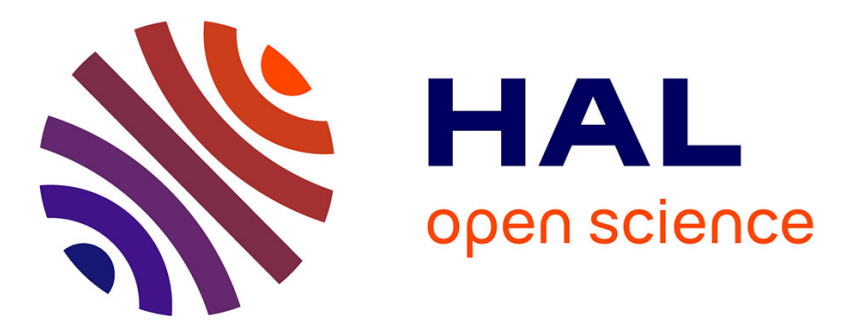

\title{
Temperature-dependent electro-mechanical actuation sensitivity in stiffness-tunable BaTiO3/polydimethylsiloxane dielectric elastomer nanocomposites.
}

\author{
Hang Zhao, Ling Zhang, Minhao Yang, Zhimin Dang, Jinbo Bai
}

\section{To cite this version:}

Hang Zhao, Ling Zhang, Minhao Yang, Zhimin Dang, Jinbo Bai. Temperature-dependent electromechanical actuation sensitivity in stiffness-tunable BaTiO3/polydimethylsiloxane dielectric elastomer nanocomposites.. Applied Physics Letters, 2015, 106 (9), pp.106(9):092904. 10.1063/1.4914012 . hal01220419

\author{
HAL Id: hal-01220419 \\ https://hal.science/hal-01220419
}

Submitted on 27 May 2020

HAL is a multi-disciplinary open access archive for the deposit and dissemination of scientific research documents, whether they are published or not. The documents may come from teaching and research institutions in France or abroad, or from public or private research centers.
L'archive ouverte pluridisciplinaire HAL, est destinée au dépôt et à la diffusion de documents scientifiques de niveau recherche, publiés ou non, émanant des établissements d'enseignement et de recherche français ou étrangers, des laboratoires publics ou privés. 


\title{
Temperature-dependent electro-mechanical actuation sensitivity in stiffness-tunable $\mathrm{BaTiO}_{3}$ /polydimethylsiloxane dielectric elastomer nanocomposites
}

\author{
Hang Zhao, ${ }^{1,2}$ Ling Zhang, ${ }^{1}$ Min-Hao Yang, ${ }^{1}$ Zhi-Min Dang, ${ }^{1,3, a)}$ and Jinbo Bai ${ }^{2}$ \\ ${ }^{1}$ Laboratory of Dielectric Polymer Materials and Devices, Department of Polymer Science and Engineering, \\ University of Science and Technology Beijing, Beijing 100083, People's Republic of China \\ ${ }^{2}$ Laboratoire de Mécanique des Sols, Structures et Matériaux, CNRS UMR8579, Ecole Centrale Paris, \\ Grande Voie des Vignes, 92290 Châtenay-Malabry Cedex, France \\ ${ }^{3}$ State Key Laboratory of Electrical Insulation and Power Equipment, Xi' an Jiaotong University, \\ Xi' an 710049, People's Republic of China
}

(Received 16 January 2015; accepted 22 February 2015; published online 3 March 2015)

\begin{abstract}
Stiffness-tunable $\mathrm{BaTiO}_{3} /$ polydimethylsiloxane (BT/PDMS) dielectric elastomer composites using dimethylsilicone oil (DMSO) as the stiffness tuner were prepared. Significant improvements in both electromechanical actuation sensitivity $(0 \%-490 \%)$ and actuation areal strain $(0 \%-350 \%)$ can be observed from BT/PDMS samples with DMSO, which should be mainly contributed to the physical swelling behavior. Under wide testing temperature range, the stiffness-tuned BT/PDMS composite exhibited an excellent thermal stability of both dielectric and mechanical performance. The electromechanical sensitivity of BT/PDMS composite can be effectively tuned higher than that of VHB 4910 acrylics via the addition of DMSO under in vivo temperature range $\left(20^{\circ} \mathrm{C}-40^{\circ} \mathrm{C}\right)$. This simple way endows PDMS-based composites the higher performance for being applied as implantable dielectric elastomer materials. C 2015 AIP Publishing LLC.
\end{abstract}

[http://dx.doi.org/10.1063/1.4914012]

Dielectric elastomer actuators (DEAs) are capable of deforming in response to an electrical field stimulation, becoming a promising branch of electroactive polymers. ${ }^{1,2}$ By virtue of their distinct functional properties from other traditional actuators, such as highly flexible, large actuation strain, high output power density, and low cost, DEAs can be potentially applied as emulational robots, haptic displays, and Braille devices. ${ }^{3,4}$ Besides these mentioned smart actuation systems, nowadays, more and more attention of DEAs has been paid to applications of the substitution of human organs and tissues, such as artificial muscles for orthotics and prosthetics, fluidic pumps for heart diseases and tunable optical lens. ${ }^{5,6}$ However, compared with in vitro applications, the DEA materials that are competent to be applied in the biological in-vivo environment should take both the electromechanical actuation performance and bio-compatibility into account. ${ }^{5}$

In the near decade, with the rapid development of DEAs field, how to maintain or improve the actuation performance under a lower driving electric field has been one of the most crucial issues. ${ }^{7}$ The general way of giving pre-stain to DEAs could increase the effective electric field, but reduce the specific output power due to the extra-mould. From the viewpoint of materials, it is necessary and reasonable to improve the intrinsic physic performance of materials. In order to evaluate the actuation performance of DEA materials, the electromechanical sensitivity $\beta$ is defined as the ratio of the relative permittivity $\varepsilon_{r}$ to the elastic modulus $Y^{2}$ Thus, the actuation strain in thickness direction is proportional to $\beta$.

\footnotetext{
${ }^{\text {a) }}$ Author to whom correspondence should be addressed. Electronic mail: dangzm@ustb.edu.cn
}

Generally, polydimethylsiloxane (PDMS) and acrylics are two main dielectric polymer elastomers that have been widely used as DEA materials. The most representative material of acrylics is the VHB 4910 (produced by 3M Co.). Due to the excellent mechanical property and high output power density, it has been deeply studied for a long time. ${ }^{8,9}$ Compared with the VHB 4910, PDMS with merits of easilyprocessing, high response speed, mechanically stable under wide-temperature range, and outstanding bio-compatibility is more suitable for operating in the biological in-vivo environment. ${ }^{10,11}$ However, pure PDMS elastomers have low intrinsic mechanical strength and relative dielectric permittivity, which determine the lower electromechanical sensitivity than that of VHB 4910. From the beginning of this century, a plenty of researches focused on increasing the permittivity of DEAs though loading high- $\varepsilon_{r}$ ceramics or conductive particles into soft polymeric matrices, to prepare the DEA composites with improved $\varepsilon_{r}{ }^{12-15}$ However, the effective enhancement of permittivity only at high loading content, where the flexibility of composite is inevitably destroyed. Thus, most of these composites failed to enhance the actuation performance effectively. In order to solve this problem, approaches of improving $\beta$ via tuning the stiffness of composites have been paid more and more attention in very recent years. ${ }^{16,17}$

The stiffness-tuned PDMS based DEA composites that are reported by our group showed improvements of both electromechanical sensitivity and output elastic energy density. ${ }^{18}$ However, comparisons of dielectric, mechanical, and electromechanical actuation performances between VHB 4910 and stiffness-tuned PDMS based composites under wide temperature range have rarely been addressed to 
date, especially under the general in-vivo temperature range $\left(\sim 20^{\circ} \mathrm{C}-40^{\circ} \mathrm{C}\right)$.

$\mathrm{BaTiO}_{3}$ (BT) with high $\varepsilon_{r}\left(\sim 10^{3}\right)$ has been widely utilized as an excellent ceramic filler. ${ }^{19}$ Nano-scale BT particles are capable of not only benefiting the $\varepsilon_{r}$ of composite but also playing an important role in strengthening polymeric matrices. Dimethylsilicone oil (DMSO) is able to be applied as an efficient stiffness tuner, which has the similar composition but lower polymerization degree than the uncrosslinked PDMS. In this letter, we investigate the BT/PDMS composites using DMSO as the stiffness tuner to significantly enhance the electro-mechanical actuation sensitivity of composites. Through tuning the stiffness, the $\beta$ of BT/PDMS composites can beat that of VHB 4910 under in vivo temperature range.

BT particles with diameter of $100 \mathrm{~nm}$ were kept constant content as $100 \mathrm{phr}$ (parts per hundreds of rubber), and a calculated amount of DMSO ranges from 0 to $100 \mathrm{phr}$ (at mass ratios to PDMS of $0 \%$ to $100 \%$ ) with the viscosity of $(0.1 \mathrm{~Pa} \cdot \mathrm{s})$ were well blended into PDMS matrix with the viscosity (90 Pa.s) through mechanical mixing. Stiffnesstunable BT/PDMS composites with typical shapes that are suitable for various tests were obtained after being cured and molded by compression at room temperature and $15 \mathrm{MPa}$ for $8 \mathrm{~h}$. Morphologies of BT/PDMS composites that before and after loading DMSO were characterized using a scanning electron microscope (SEM) (Hitachi S-4700). The dielectric properties were characterized as a function of frequency (from $1 \mathrm{kHz}$ to $1 \mathrm{MHz}$ ) and temperature (from $-20^{\circ} \mathrm{C}$ to $90^{\circ} \mathrm{C}$ ) using an impedance analyzer (Agilent 4294A) and supporting temperature chamber (Espec SU-261). The elastic modulus at room temperature was tested by an electric tester (Instron 1185). The temperature-dependent mechanical property was investigated by dynamic mechanical analysis (DMA) (TA Q800) in uniaxial tension mode at $1 \mathrm{~Hz}$ and the temperature range of $-20{ }^{\circ} \mathrm{C}-90^{\circ} \mathrm{C}$ was scanned at a heating rate of $1 \mathrm{~K} / \mathrm{min}$.

SEM images of BT/PDMS composites before and after loading DMSO are presented in the inset of Fig. 1(a). Due to the similar chemical composition and extraordinary miscibility, no obvious interface between DMSO and PDMS matrix can be observed. Moreover, nano-BT particles are well dispersed and distributed in PDMS matrix, indicating that the way of chemical-free mechanical mixing is potential to make this stiffness-tunable DEA composite scalable from laboratory to industrial scale.

During the actuation measurement, nano-carbon black powders were coated onto two surfaces of sample film with thickness of $150 \mu \mathrm{m}$, in order to connect a direct-current electric field with the composite film. As shown in Fig. 1(a), the actuation areal strain displays positive correlations to not only the driving electrical field but also the DMSO concentration in BT/PDMS composites. It is worth to note that an improvement of more than $350 \%$ of actuation strain can be achieved from the sample with $100 \mathrm{phr}$ DMSO under the driving electric field as low as $20 \mathrm{~V} / \mu \mathrm{m}$.

The addition of DMSO is able to effectively modify both the dielectric and mechanical performance of composite, which could be responsible for the dramatic improvement of actuation stain (See Fig. 1(b)). On one hand,
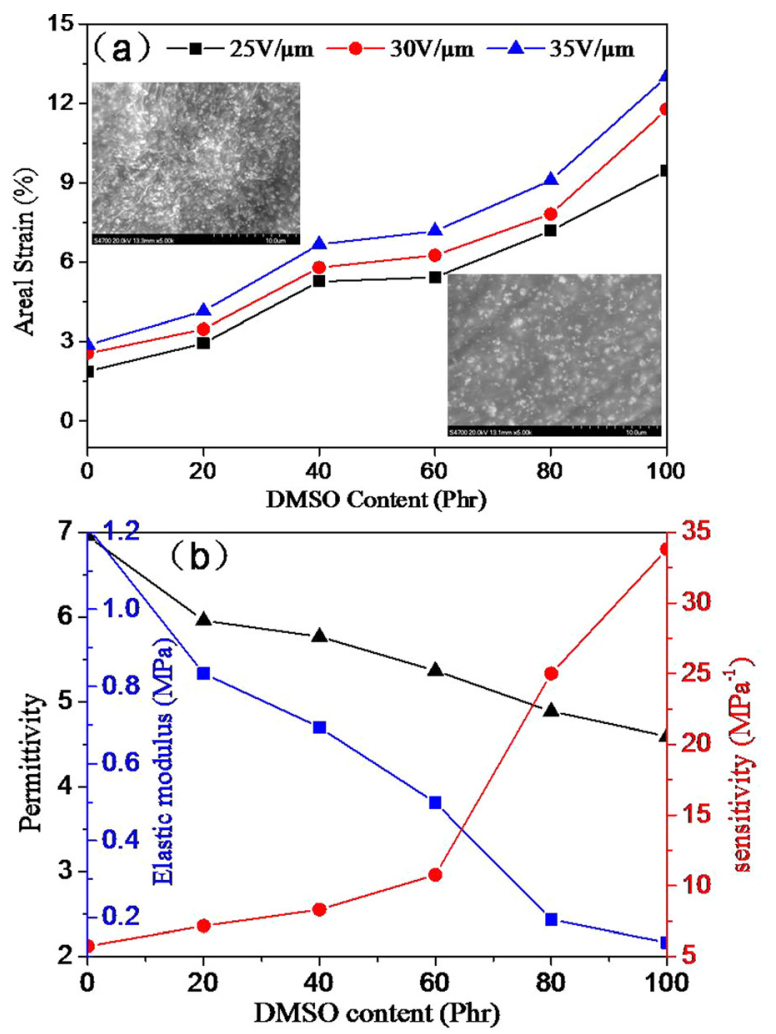

FIG. 1. Influences of added DMSO on BT/PDMS composites. (a) The relationship between actuation areal strain and DMSO content at low electric fields: $20 \mathrm{~V} / \mu \mathrm{m}, 25 \mathrm{~V} / \mu \mathrm{m}$, and $30 \mathrm{~V} / \mu \mathrm{m}$. Insets show that typical SEM images of fractured surface of the BT/PDMS without (left) and with (right) $100 \mathrm{phr}$ of DMSO. (b) Relative permittivity, elastic modulus, and electromechanical sensitivity vs. DMSO content curves.

compared with the unplasticized BT/PDMS composite, the added $100 \mathrm{Phr}$ DMSO dilutes the concentration of BT to the half of its original volume fraction (the difference can be distinguished between SEM images), which gives rise to the corresponding $34 \%$ decrease of $\varepsilon_{r}$ of composite. On the other hand, a greater drop ( $\sim 89 \%)$ of the elastic modulus of composites can be observed under room temperature, which ascribes to the physical swelling effect of DMSO to molecular chains of PDMS. The swelling effect is the physical process of that the conformation of polymer chains can change from highly randomly curled state to partly stretched state through mixing the solvents with low degree of polymerization. It can induce a decrease to modulus which is in inverse proportion to the cube root of the swelling ratio. ${ }^{20}$ As an extraordinary plasticizer to PDMS, the added DMSO could effectively weaken the intermolecular interactions, increase the mobility of macromolecular chains, and decrease the elastic modulus of composites. Thus, the integrative effect is that the electromechanical sensitivity is significantly enhanced by $490 \%$ with DMSO content from 0 to $100 \%$.

To investigate influences of temperature on dielectric performance, Figs. 2(a)-2(c) demonstrate variations of relative permittivity as a function of frequency and temperature of BT/PDMS composite, BT/PDMS composite with $100 \mathrm{phr}$ DMSO, and VHB 4910 acrylics, respectively. In contrast with acrylics, the relative permittivity of PDMS-based composites is susceptible to their internal compositions. Namely, the permittivity of the BT/PDMS composites is higher than 

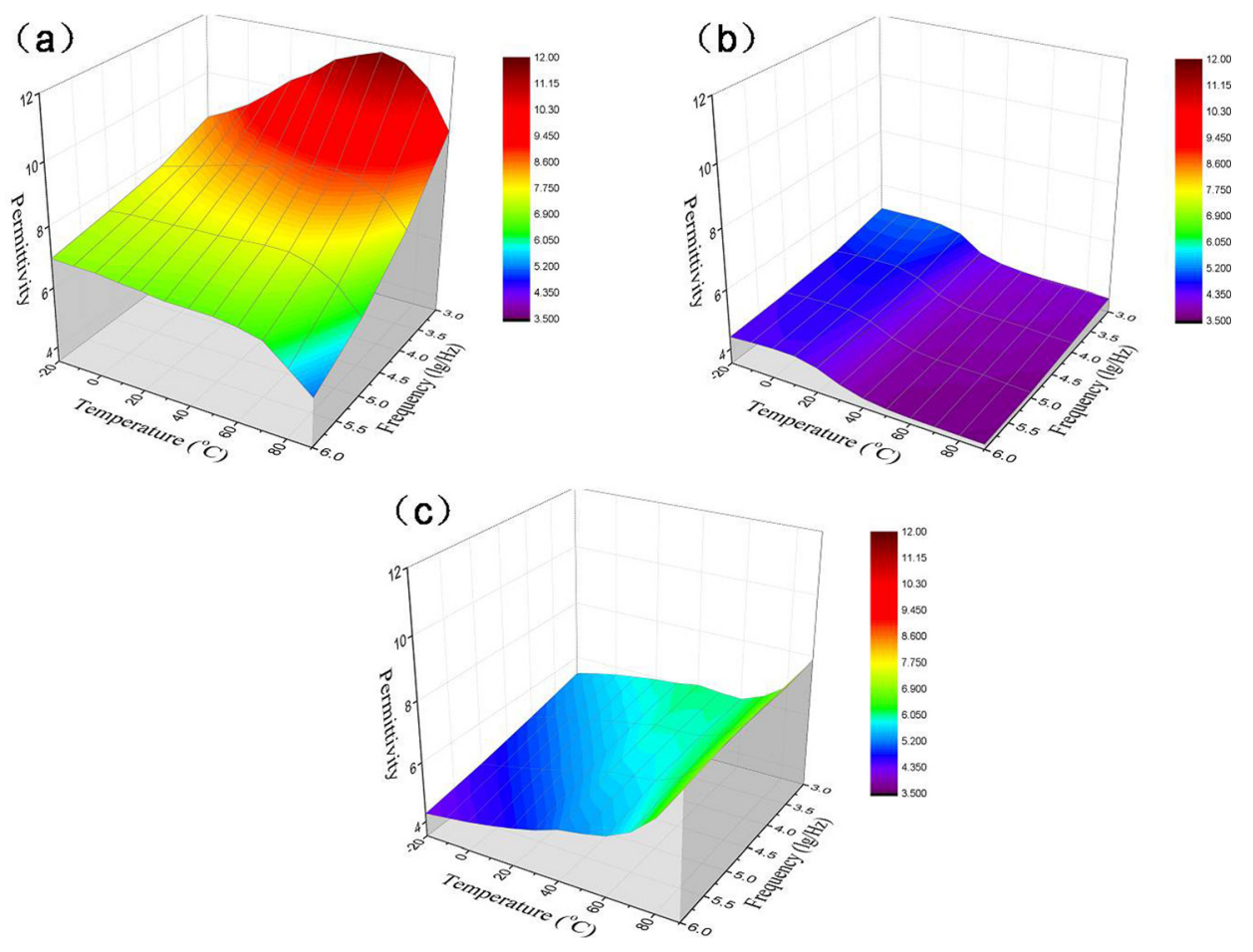

FIG. 2. Dependence of relative permittivity on frequency and temperature for (a) BT/PDMS composite; (b) BT/ PDMS composite with $100 \mathrm{phr}$ of DMSO; (c) VHB 4910 acrylics.

that of acrylics due to the addition of BT. However, the additional 100 phr DMSO leads to an inverse effect.

Besides their different magnitudes, notable distinctions of response of permittivity to temperature can be observed from colormaps of these 3 samples. For the Fig. 2(a), $\varepsilon_{r}$ increases with enhancing temperature from $-20^{\circ} \mathrm{C}$ to $60^{\circ} \mathrm{C}$. It may result from the expansion of free volume of PDMS molecular chains, which makes dipoles in the BT/ PDMS composite orientate easily under electrical field; With the increase in temperature, the thermal agitation of dipoles should be responsible for the following drop of $\varepsilon_{r}{ }^{21}$ However, the added DMSO could give rise to the preexpansion of free volume of PDMS molecular chains, according to the swelling theory. Therefore, the main drop tendency of temperature-dependent permittivity in Fig. 2(b) could attribute to the drastic thermal agitation of dipoles with the increase in temperature. In addition, as there is no additional filler loaded into the acrylic film, the positive temperature-response of $\varepsilon_{r}$ could be mainly ascribed to the free volume expansion with increasing temperature (see Fig. 2(c)).

As we mentioned before, both the electromechanical sensitivity and output elastic energy density are determined by not only dielectric properties but also mechanical properties. Different from the uniaxial tension under room temperature, the temperature-dependent elastic modulus of BT/PDMS composite, BT/PDMS composite with $100 \mathrm{phr}$ DMSO, and VHB 4910 acrylics were characterized by DMA. As shown in Fig. 3(a), be similar with the static elastic modulus, dynamic storage modulus displays a reduction after loading DMSO into BT/PDMS composite under tested wide temperature range. However, under the same temperature range, the acrylic shows a distinctively strong drop of storage modulus (from $200 \mathrm{MPa}$ to $0.2 \mathrm{MPa}$ ), which could be explained by the corresponding relaxation peak of acrylics from the plots of loss tangent to temperature (see
Fig. 3(b)). In fact, both the drastic drop of storage modulus and the significant loss peak may correspond to the glass transition of VHB 4910 acrylics.
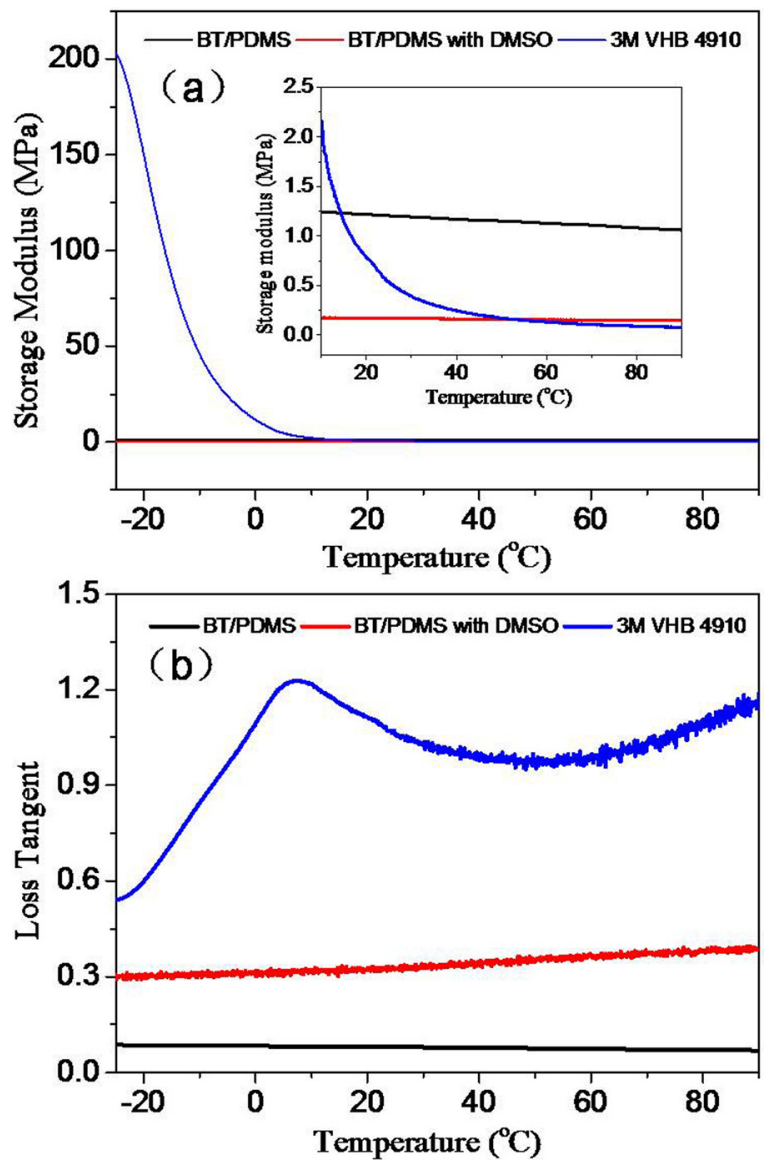

FIG. 3. Typical DMA curves of BT/PDMS composite, BT/PDMS composite with $100 \mathrm{phr}$ of DMSO, and VHB 4910 acrylics at $1 \mathrm{~Hz}$. (a) Storage modulus; (b) Mechanical loss tangent. 
However, due to the typical helical structure of Si-O chains, there are the weak intermolecular interactions among these chains, moreover, which can be further reduced through loading DMSO. It is notable that both the high bonding energy $(446 \mathrm{~kJ} / \mathrm{mol})$ and long bond length $(1.64 \AA)$ of "Si-O-" backbone could contribute to the temperatureinsensitive intermolecular interactions, which endows PDMS with relatively steady thermal-performances. ${ }^{22,23}$ Generally, the viscoelasticity of elastomers can be evaluated from the loss tangent results of DMA test. Compared with the PDMSbased composites with inert di-methyl as lateral groups, the lateral groups of acrylics (such as ester group and alkyl chain) may have larger volumes and steric hindrances. Thus, the distinctly high viscosity can be measured from the VHB 4910 sample, which is in accordance with its frequently reported low actuation response. Although the loss tangent of BT/PDMS composite displays a slight enhancement after adding the DMSO, it still maintains in a relatively low level and shows a stable mechanical performance under wide temperature range.

Similar to the $\beta$ at room temperature, the temperaturedependent electromechanical sensitivity $\beta_{T}$ could be defined as the ratio between the relative permittivity at $1 \mathrm{kHz}$ and the storage modulus under wide temperature range from $-20^{\circ} \mathrm{C}$ to $90^{\circ} \mathrm{C}$. Fig. 4 presents a comparison of temperaturedependent electromechanical sensitivities of mentioned three samples. Due to the intrinsic mechanical performance of VHB 4910, its electromechanical sensitivity spans over 4 orders of magnitudes. On the contrary, PDMS-based composites show relatively steady $\beta_{T}$, owing to their excellent thermal stability. It is noteworthy that the addition of DMSO makes the mean value of $\beta_{T}$ under tested temperature range increased by $225 \%$. Particularly, testing temperature range can be divided into three individual stages: I. from -20 to $21{ }^{\circ} \mathrm{C}$; II. from $21^{\circ} \mathrm{C}$ to $42{ }^{\circ} \mathrm{C}$; and III. from $42{ }^{\circ} \mathrm{C}$ to $90^{\circ} \mathrm{C}$ (see the inset of Fig. 4). According to the magnitudes of $\beta$, there would be an optimally-suitable DEA material in each stage. Namely, the investigated three samples have their preferable operation temperature range.

For the stage I, due to the much lower glass transition temperature $\left(T_{g}\right)$ of PDMS than that of acrylics, BT/PDMS composites show the higher and more stable $\beta_{T}$, and become

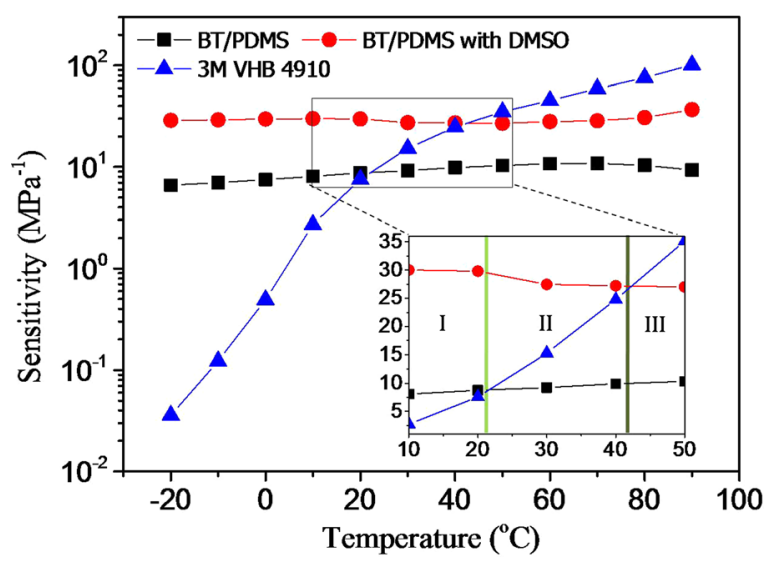

FIG. 4. Dependence of electromechanical sensitivity on temperature for BT/ PDMS composite, BT/PDMS composite with $100 \mathrm{phr}$ of DMSO and VHB 4910 acrylics. Inset demonstrates the defined three stages. preferable DEA materials when operating under low temperature range. When the operation temperature is over than $42^{\circ} \mathrm{C}$ (the stage III), although acrylics possesses high loss tangent and viscosity, it still demonstrates higher $\beta_{T}$ and flexibility than PDMS-based composites. The most noteworthy part is stage II, where the temperature range (from 21 to $42^{\circ} \mathrm{C}$ ) corresponds to the biological in-vivo environment. On one hand, DMSO is loaded into the BT/PDMS composite leads to a significant improvement of $\beta_{T}$ compared with that of both its pure counterpart and even VHB 4910, which makes the stiffness-tuned BT/PDMS composite as the optimal choice of DEA material in this stage. On the other hand, the operation temperature range (limits of stage II) can be well controlled by tuning the DMSO content of PDMS matrix.

Therefore, the simple way that adding DMSO to tune the stiffness of composites can effectively improve the $\beta$ of PDMS-based composites. Moreover, it also provides another option for DEA materials in biological applications under the in vivo temperature range.

In conclusion, the stiffness-tunable BT/PDMS dielectric elastomer composites using DMSO as stiffness tuner were fabricated by a chemical-free mechanical mixing approach. The addition of DMSO was capable of making a small reduction of relative permittivity and a much greater drop of elastic modulus. The combined effect leads to that both electromechanical sensitivity and actuation strain can be increased by $490 \%$ and $350 \%$, respectively, for the sample BT/PDMS composite with $100 \mathrm{phr}$ of DMSO. Moreover, the stiffness-tuned BT/PDMS composite demonstrates stable thermal responses of both dielectric and mechanical performance under widely testing temperature range. The electromechanical actuation sensitivity of BT/PDMS composite can be effectively tuned to be higher than that of the general DEA material, VHB 4910 acrylics, under the in-vivo temperature range of $21-40^{\circ} \mathrm{C}$. This simple approach of tuning stiffness of composite using DMSO makes PDMS-based composites more potential and suitable for applications in highly sensitive, biocompatible, and implantable DEA materials.

This work was financially supported by NSF of China (Grant Nos. 51425201 and 51377010), Ministry of Education of China through Doctor Project (Grant No. 20130006130002), the National Basic Research Program of China (973 Program) (Grant No. 2014CB239503), and State Key Laboratory of Electrical Insulation and Power Equipment (EIPE14204).

${ }^{1}$ P. Brochu and Q. B. Pei, Macrom. Rapid Commun.31, 10 (2010).

${ }^{2}$ A. O'Halloran, F. O'Malley, and P. McHugh, J. Appl. Phys. 104, 071101 (2008).

${ }^{3}$ G. Frediani, D. Mazzei, D. E. De Rossi, and F. Carpi, Front. Bioeng. Biotechnol. 2, 31 (2014).

${ }^{4}$ C. A. Hanley, Y. K. Gun'ko, G. Frediani, and F. Carpi, Smart Mater. Struct. 23, 015009 (2014).

${ }^{5}$ F. Carpi, R. Kornbluh, P. Sommer-Larsen, and G. Alici, Bioinspiration Biomimetics 6, 045006 (2011).

${ }^{6}$ F. Carpi, G. Frediani, S. Turco, and D. De Rossi, Adv. Funct. Mater. 21, 4152 (2011).

${ }^{7}$ F. Carpi, S. Bauer, and D. De Rossi, Science. 330, 1759 (2010). 
${ }^{8}$ S. M. Ha, W. Yuan, Q. Pei, R. Pelrine, and S. Stanford, Adv. Mater. 18, 887 (2006).

${ }^{9}$ R. Pelrine, R. Kornbluh, Q. B. Pei, and J. Joseph, Science. 287, 836 (2000).

${ }^{10}$ M. Molberg, D. Crespy, P. Rupper, F. Nüesch, J.-A. E. Månson, C. Löwe, and D. M. Opris, Adv. Funct. Mater. 20, 3280 (2010).

${ }^{11}$ D. M. Opris, M. Molberg, C. Walder, Y. S. Ko, B. Fischer, and F. A. Nüesch, Adv. Funct. Mater. 21, 3531 (2011).

${ }^{12}$ C. Huang, Q. M. Zhang, G. deBotton, and K. Bhattacharya, Appl. Phys. Lett. 84, 4391 (2004).

${ }^{13}$ H. Zhao, Y. J. Xia, Z. M. Dang, J. W. Zha, and G. H. Hu, J. Appl. Polym. Sci. 127, 4440 (2013).

${ }^{14}$ D. Wang, L. Zhang, L. Zhang, J.-W. Zha, H. Yu, S. Hu, and Z.-M. Dang, Appl. Phys. Lett. 104, 242903 (2014).

${ }^{15}$ Z. M. Dang, J. K. Yuan, J. W. Zha, T. Zhou, S. T. Li, and G. H. Hu, Prog. Mater. Sci. 57, 660 (2012).
${ }^{16}$ H. C. Nguyen, V. T. Doan, J. Park, J. C. Koo, Y. Lee, J.-d. Nam, and H. R. Choi, Smart Mater. Struct. 18, 015006 (2009).

${ }^{17}$ F. Galantini, F. Carpi, and G. Gallone, Smart Mater. Struct. 22, 104020 (2013).

${ }^{18}$ H. Zhao, D.-R. Wang, J.-W. Zha, J. Zhao, and Z.-M. Dang, J. Mater. Chem. A. 1, 3140 (2013).

${ }^{19}$ Z.-M. Dang, H.-P. Xu, and H.-Y. Wang, Appl. Phys. Lett. 90, 012901 (2007).

${ }^{20}$ L. R. G. Treloar, The Physics of Rubber Elasticity (Clarendon Press, Oxford, 1975).

${ }^{21}$ B.-H. Fan, J.-W. Zha, D.-R. Wang, J. Zhao, and Z.-M. Dang, Appl. Phys. Lett. 100, 092903 (2012).

${ }^{22}$ H. Cochrane and C. S. Lin, Rubber Chem. Technol. 66, 48 (1993).

${ }^{23}$ J. Biggs, K. Danielmeier, J. Hitzbleck, J. Krause, T. Kridl, S. Nowak, E. Orselli, X. N. Quan, D. Schapeler, W. Sutherland, and J. Wagner, Angew. Chem. Int. Ed. 52, 9409 (2013). 